\title{
ANÁLISE DE SUSPENSÕES DE ARGILAS POR ESPECTROMETRIA DE EMISSÃo ÓPTICA COM PLASMA INDUZIDO COM CONFIGURAÇÃO AXIAL
}

\author{
Cíntia S. Silva e Joaquim A. Nóbrega* \\ Departamento de Química, Universidade Federal de São Carlos, CP 676, 13560-970 São Carlos - SP \\ Telma Blanco \\ Centro de Caracterização e Desenvolvimento de Materiais, Universidade Federal de São Carlos - Universidade Estadual Paulista \\ Júlio de Mesquita Filho, CP 147, 13560-970 São Carlos - SP
}

Recebido em 23/1/02; aceito em 17/4/02

\begin{abstract}
CLAY SLURRIES ANALYSIS USING INDUCTIVELY COUPLED PLASMA OPTICAL EMISSION SPECTROMETRY WITH AXIAL VIEW CONFIGURATION. The goal of this study was to evaluate the feasibility of direct introduction of clay slurries in an inductively coupled plasma optical emission spectrometer with axial view configuration. Calibration was performed using a certified reference material with a mean particle size of $13 \mu \mathrm{m}$ (IPT-42) and the analytical curve was applied for quantification of two others reference materials (IPT-28 and IPT-32) and four samples. It was demonstrated that the analytical curve thus obtained was not completely suitable for IPT-28 and samples due to different mineralogical phases determined by X-ray diffraction. After considering this effect, it was possible for most elements to obtain results in agreement with certified values or with values obtained by a conventional technique at a $95 \%$ confidence level. It was demonstrated that the ICP-OES with axial view configuration did not present any incompatibility with the direct introduction of a complex inorganic suspension.
\end{abstract}

Keywords: clay slurries; inductively coupled plasma optical emission spectrometry; axial view configuration.

\section{INTRODUÇÃO}

A análise química de materiais inorgânicos geralmente envolve uma etapa de conversão da amostra sólida em uma solução representativa, pois as técnicas espectroanalíticas freqüentemente utilizadas para quantificação dos analitos geralmente envolvem a introdução de amostras como soluções ${ }^{1}$. Dependendo do tipo de material, essa etapa é morosa e requer o uso de ácidos concentrados sob elevadas temperaturas. Um procedimento alternativo é a introdução da amostra na forma de uma suspensão.

A análise direta de suspensões por espectrometria de absorção atômica com forno de grafite está bem estabelecida pois essa técnica não envolve a introdução da amostra via nebulização pneumática e efeitos causados por tamanho de partículas são menos pronunciados ${ }^{2}$.

Outra alternativa é a introdução de suspensões em espectrômetros de emissão óptica com plasma induzido (ICP-OES) utilizando nebulizadores adequados para trabalhar com alta concentração de sólidos, tais como nebulizadores tipo Babington e com ranhura em $\mathrm{V}^{3}$. Essa estratégia gerou uma série de procedimentos adequados, conforme se pode constatar no artigo de revisão publicado por Ebdon et al. ${ }^{4}$. Entretanto, devido aos processos que ocorrem na conversão da suspensão em um aerossol durante a introdução da amostra e aos processos de atomização-excitação no plasma, a análise direta de suspensões por ICP-OES é criticamente afetada pela distribuição de tamanho de partículas. Partículas grandes não atingem o plasma ou não são eficientemente convertidas em átomos excitados durante o curto tempo de residência no plasma. O tamanho de partícula ideal depende das características do sistema de introdução de amostra, do comportamento termoquímico das partículas e das propriedades do plasma considerando-se processos de transferência de calor e tempo de residência. De acordo com Broekaert et al. ${ }^{5}$ a análise de suspensões de materiais cerâmicos requer partículas com tamanho inferior

*e-mail: djan@terra.com.br a $5 \mu \mathrm{m}$ para evitar efeitos pronunciados sobre os processos de nebulização e atomização-excitação. Ebdon et al. ${ }^{4}$ também destacaram que intensidades de sinais de emissão gerados por suspensões e soluções somente são equivalentes para suspensões contendo partículas inferiores a $6 \mu \mathrm{m}$ e, conseqüentemente, a calibração somente pode ser efetuada com soluções analíticas se as suspensões das amostras apresentarem partículas inferiores a esse limite. Anteriormente, Fuller et al. ${ }^{6}$ já tinham destacado esse mesmo valor limite. Pode-se supor que esse valor esteja fortemente influenciado pelas características do sistema de introdução de amostras, pois é conhecido que somente partículas inferiores a $5 \mu \mathrm{m}$ são eficientemente transportadas quando soluções são pneumaticamente introduzidas ${ }^{7}$.

A quase totalidade dos trabalhos sobre a introdução de suspensões em ICP-OES's envolvem sistemas com configuração radial ${ }^{4}$. O comportamento de suspensões em ICP-OES's com configuração axial é praticamente desconhecido ${ }^{8}$ e entre os aspectos que precisam ser elucidados destacam-se os efeitos das partículas sólidas sobre a interface, a possibilidade de interferências espectrais mais intensas e alterações do tempo médio de residência das partículas no plasma. Apesar da visão geral que espectrômetros com configuração axial deveriam ser preferencialmente aplicados para matrizes menos complexas, estudos recentes mostram que as interferências causadas pela matriz não são significativas quando a interface envolve um contrafluxo de argônio introduzido frontalmente ao plasma ${ }^{9,10}$. Assim, terras raras foram determinados com sucesso em fezes de carneiros ${ }^{9} \mathrm{e}$ suspensões de cimento foram diretamente analisadas ${ }^{10}$.

O comportamento de suspensões de argila em ICP-OES com configuração axial ainda não é conhecido. Em equipamento com configuração radial, Laird et al. ${ }^{11}$ demonstraram a viabilidade da análise direta de suspensões de argilas para amostras com partículas inferiores a 2 $\mu \mathrm{m}$. Esses autores adotaram calibração com soluções aquosas e mostraram que a recuperação de todos os analitos é criticamente dependente do tamanho de partículas, observando-se um decréscimo conforme esse parâmetro aumentou de $<2 \mu$ m para a faixa de 10 a $45 \mu$ m. 
O objetivo deste trabalho foi a introdução de suspensões de argilas em ICP-OES com configuração axial e a avaliação da viabilidade desse procedimento para a análise química rápida desse material.

\section{PARTE EXPERIMENTAL}

\section{Instrumentação}

Todas as determinações foram feitas usando um ICP-OES VISTA AX simultâneo com configuração axial (Varian, Mulgrave, Austrália). Esse equipamento contém um sistema óptico e um detector que torna possível obter um espectro em duas dimensões no qual sinais de emissão são separados em função do comprimento de onda e da ordem espectral. O sistema de introdução de amostras é constituído por uma câmara de nebulização do tipo Sturman-Masters de politetrafluoretileno (PTFE) e um nebulizador com ranhura em V que possibilita a introdução de suspensões. Os parâmetros operacionais e as linhas de emissão estão apresentados na Tabela 1.

Tabela 1. Parâmetros operacionais do ICP-OES com configuração axial para análise de amostras de suspensões de argila

\section{Parâmetros}

\begin{tabular}{lc}
\hline Gerador de rádio frequiência (MHz) & 40 \\
Potência Incidente (kW) & 1,2 \\
Vazão do gás do plasma (L/min) & 15,0 \\
Vazão do gás auxiliar (L/min) & 1,5 \\
Tempo de estabilização (s) & 0,7 \\
Tempo de leitura (s) & 15 \\
Replicatas & 3
\end{tabular}

Câmara de nebulização Sturman-Master

Nebulizador com ranhura em $\mathrm{V}$

Linhas de emissão $(\lambda / \mathrm{nm})$

\author{
Al I 394,405 \\ Ca II 396,486 \\ Fe II 238,203 \\ K I 766,498 \\ Mg II 280,265 \\ Na I 589,587 \\ Si I 288,158 \\ Ti II 334,939 \\ P I 213,618
}

A distribuição do tamanho de partículas foi determinada usando o equipamento LS 230 (Coulter Co, Miami, FL, EUA), que possibilita medidas de tamanho de partículas entre 0,04 e $2000 \mu \mathrm{m}$. Esse equipamento opera com difração de radiação laser baseada nos modelos de Fraunhofer e $\mathrm{Mie}^{12}$.

Os espectros de difração de raio-X foram obtidos utilizando um equipamento Siemens D 5000 (Munique, Alemanha) usando um tubo de raio X de cobre operando a $40 \mathrm{kV}$ e $40 \mathrm{~mA}$, e um filtro de Ni.

Para comparação dos resultados das amostras comerciais de argila, algumas medidas foram feitas em um espectrômetro de fluorescência de raios X Phillips PW-1480 (Holanda), usando um tubo revestido com filamento de cobre operando a $45 \mathrm{kV}$ e $75 \mathrm{~mA}$.

Um banho de ultra-som USC (Unique1400, SP, Brasil) foi usado para desagregar as suspensões.

\section{Amostras e reagentes}

Ácido nítrico grau analítico (Mallinckrodt, México) foi utilizado para preparar as suspensões das amostras de argila.
As soluções de referência para a calibração do ICP-OES foram preparadas a partir de soluções estoque multielementares (SPEX, Metuchen, NJ, EUA)

Materiais de referência certificados do Instituto de Pesquisas Tecnológicas do Estado de São Paulo (IPT) foram utilizados para avaliar a exatidão e para calibrar o equipamento: IPT-28 (argila Pará), IPT-32 (argila plástica Saracura) e IPT-42 (argila São Simão).

Todos os reagentes utilizados neste estudo foram de grau analítico e foi usada água purificada pelo sistema Mili-Q ${ }^{\circledR}$ (Milipore, Bedford, MA, EUA).

Também foram utilizadas quatro amostras de argilas obtidas juntas ao Centro de Caracterização e Desenvolvimento de Materiais.

\section{Preparo de suspensões e calibração}

Os materiais de referência certificados e as amostras comerciais de argila foram preparadas usando um moinho vibratório de ágata por $20 \mathrm{~min}$, seguida de moagem manual por 5 a $10 \mathrm{~min}$, usando almofariz e pistilo de ágata.

Duas estratégias foram utilizadas para a calibração. A primeira baseou-se na suspensão de massas crescentes de material de referência certificado em solução $10 \%$ v/v de $\mathrm{HNO}_{3}$. Outra estratégia envolveu o preparo de suspensões nesse mesmo meio ácido a partir de massas constantes de diferentes materiais de referência certificados. Nesse caso, massas de $80 \mathrm{mg}$ de cada material de referência certificado foram suspensas em $100 \mathrm{~mL}$ de solução $10 \%$ v/v $\mathrm{HNO}_{3}$.

\section{RESULTADOS E DISCUSSÃO}

O preparo de suspensões e a introdução dessas suspensões por nebulização pneumática em ICP-OES é criticamente dependente do tamanho de partículas. Assim, os primeiros experimentos envolveram a caracterização da distribuição do tamanho de partículas para o material de referência certificado IPT-42, selecionado para preparo das suspensões para calibração do equipamento. A medida de distribuição de tamanho de partículas indicou que as partículas se situam no intervalo de 1 a $50 \mu \mathrm{m}$, com tamanho médio de $13 \mu \mathrm{m}$. Apenas $10 \%$ das partículas tinham tamanho superior a 14,6 $\mu \mathrm{m}$. O tamanho de partícula médio é superior ao tamanho de partícula ideal para introdução de suspensões por nebulização pneumática. Conseqüentemente, os efeitos sobre os processos de nebulização e atomizaçãoexcitação são severos e impedem que a quantificação dos analitos nas suspensões seja feita a partir da calibração com soluções aquosas.

Dessa forma, o material de referência certificado IPT-42 foi utilizado para calibração do equipamento visando a análise química dos materiais IPT-28 e IPT-32, que são duas outras argilas certificadas com composição química e distribuição de tamanho de partículas similares. Os resultados obtidos estão apresentados na Tabela 2. Para a argila IPT-32 não há diferença entre teores determinados e teores certificados para um nível de confiança de 95\%. Entretanto, para esse mesmo nível de confiança ocorreram diferenças estatisticamente relevantes para a determinação do elemento Si para a argila IPT-28. Esse resultado discordante para esse material foi inesperado, considerando-se as similaridades de composição química e distribuição de tamanho de partículas entre o material usado para preparo das suspensões de calibração e a argila IPT-28. Então, visando uma melhor compreensão da discrepância observada, caracterizouse a estrutura mineralógica desses materiais por difração de raios $\mathrm{X}$. Todos os materiais de referência contêm a fase caulinita $\left(\mathrm{Al}_{2} \mathrm{O}_{3} \cdot 2\right.$ $\left.\mathrm{SiO}_{2} \cdot \mathrm{H}_{2} \mathrm{O}\right)$. A fase morfológica quartzo $\left(\mathrm{SiO}_{2}\right)$ está ausente no material IPT-28 e presente nas demais argilas. Isso implica que a calibração feita com material contendo diferentes fases não é válida 
Tabela 2. Teores percentuais $(\% \mathrm{~m} / \mathrm{m})$ determinados e certificados para os materiais de referência certificados IPT-28 (argila Pará) e IPT-32 (argila Plástica Saracura). Média (desvio padrão) $(\mathrm{n}=3)$

\begin{tabular}{ccccc}
\hline Óxido & $\begin{array}{c}\text { Determinado } \\
\text { IPT-28 }\end{array}$ & $\begin{array}{c}\text { Certificado } \\
\text { IPT-28 }\end{array}$ & $\begin{array}{c}\text { Determinado } \\
\text { IPT-32 }\end{array}$ & $\begin{array}{c}\text { Certificado } \\
\text { IPT-32 }\end{array}$ \\
\hline $\mathrm{SiO}_{2}$ & $63,8(6,1)$ & 45,1 & $55,5(2,3)$ & 51,8 \\
$\mathrm{Al}_{2} \mathrm{O}_{3}$ & $46,3(3,3)$ & 37,6 & $36,1(0,9)$ & 28,5 \\
$\mathrm{Fe}_{2} \mathrm{O}_{3}$ & $0,84(0,05)$ & 0,83 & $4,51(0,12)$ & 3,46 \\
$\mathrm{TiO}_{2}$ & $3,48(0,22)$ & 2,04 & $1,64(0,08)$ & 1,49 \\
$\mathrm{P}_{2} \mathrm{O}_{3}$ & $0,23(0,02)$ & 0,15 & $0,15(0,02)$ & 0,13 \\
$\mathrm{CaO}$ & $0,05(0,01)$ & 0,09 & $0,06(0,01)$ & 0,17 \\
$\mathrm{MgO}$ & $0,03(0,004)$ & 0,04 & $0,46(0,01)$ & 0,39 \\
$\mathrm{Na}_{2} \mathrm{O}$ & $0,01(0,001)$ & 0,02 & $0,15(0,04)$ & 0,16 \\
$\mathrm{~K}_{2} \mathrm{O}$ & $0,09(0,03)$ & 0,03 & $0,53(0,03)$ & 0,80 \\
\hline
\end{tabular}

para a análise de amostras com diferentes estruturas mineralógicas. Diferentes fases mineralógicas terão distintos comportamentos térmicos, o que afetará as condições de atomização-excitação considerando-se o reduzido tempo de residência das partículas no plasma. Ressalta-se que efeitos causados por fases mineralógicas também são observados na digestão ácida convencional de materiais inorgânicos, sendo que esses efeitos são mais intensos quando compostos de silício estão envolvidos ${ }^{13}$.

Considerando-se esses resultados e sabendo-se que, de acordo com a análise por difração de raios $\mathrm{X}$, as amostras 1 a 4 contêm as fases mineralógicas quartzo, moscovita $\left(\mathrm{KAl}_{2} \mathrm{O}_{3} \cdot 4 \mathrm{SiO}_{2} \cdot \mathrm{H}_{2} \mathrm{O}\right)$, hematita $\left(\mathrm{Fe}_{2} \mathrm{O}_{3}\right)$ e montemorilonita $\left(\mathrm{Al}_{2} \mathrm{O}_{3} .4 \mathrm{SiO}_{2} . \mathrm{H}_{2} \mathrm{O}\right)$ pode-se concluir que a calibração com o material de referência certificado IPT-42 não gerará uma curva analítica adequada para essas amostras. Por outro lado, considerando-se que todas essas amostras têm a mesma procedência e similares composições químicas, optou-se por utilizar a amostra 4 para preparo das suspensões e obtenção da curva analítica. Os resultados obtidos estão apresentados na Tabela 3, que também contém os resultados obtidos por fluorescência de raios X. Com exceção do elemento Si, observa-se uma concordância de resultados em um nível de confiança de 95\%. Resultados preliminares confirmaram a impossibilidade de obter esse mesmo nível de exatidão, quando a calibração foi efetuada com suspensões preparadas a partir do material IPT-42.

Pode-se concluir que a análise direta de suspensões de argilas exige a calibração com suspensões preparadas a partir de material contendo composição química e estrutura mineralógica similar às amostras que serão analisadas. Isso implica que o analista necessita de informações sobre os materiais de referência certificados e as amostras que geralmente não estão disponíveis e não podem ser obtidas rapidamente. A tentativa de utilizar uma curva analítica de calibração obtida a partir de suspensões preparadas utilizando diferentes materiais, que aparentemente minimizaria efeitos causados pela composição química e estrutura mineralógica ${ }^{14}$, não foi eficaz para gerar resultados mais exatos.

Uma outra alternativa seria aperfeiçoar a etapa de moagem, visando a obtenção de sub-amostras com menores tamanhos médios de partículas. Apesar de viável, essa estratégia pode implicar em procedimentos morosos devido à longa etapa de moagem e aumenta a possibilidade de contaminação das amostras. Além disso, a estabilização de suspensões com partículas diminutas é difícil e requer uma cuidadosa escolha de agentes surfactantes, que estabilizem a suspensão e evitem a aglomeração de partículas.

Pode-se concluir que a análise de argilas a partir de suspensões por ICP-OES é viável, porém a aplicação em rotina pode, em algumas situações, ser limitada pela dificuldade para calibração. Entretanto, esse aspecto pode ser superado conhecendo-se as características químicas e mineralógicas das amostras.

Tabela 3. Análise química de amostras de argilas: teores obtidos $(\% \mathrm{~m} / \mathrm{m})$ a partir da análise direta de suspensões e teores obtidos por fluorescência de raios X (XRF). Média (desvio padrão) $(\mathrm{n}=3)$

\begin{tabular}{|c|c|c|c|c|c|c|}
\hline Óxido & $\begin{array}{c}\text { A1 } \\
\text { Suspensão }\end{array}$ & XRF & $\begin{array}{c}\text { A2 } \\
\text { Suspensão }\end{array}$ & XRF & $\begin{array}{c}\text { A3 } \\
\text { Suspensão }\end{array}$ & XRF \\
\hline $\mathrm{SiO}_{2}$ & $56,6(2,8)$ & 68,6 & $63,1(2,4)$ & 66,7 & $66,3(5,7)$ & 69,4 \\
\hline $\mathrm{Al}_{2} \mathrm{O}_{3}$ & $13,3(0,4)$ & 15,5 & $12,7(0,6)$ & 14,8 & $13,8(0,8)$ & 14,2 \\
\hline $\mathrm{Fe}_{2}^{2} \mathrm{O}_{3}$ & $5,33(0,05)$ & 5,47 & $4,58(0,19)$ & 4,89 & $5,73(0,23)$ & 5,38 \\
\hline $\mathrm{TiO}_{2}$ & $0,44(0,06)$ & 0,64 & $0,57(0,03)$ & 0,59 & $0,44(0,04)$ & 0,64 \\
\hline $\mathrm{CaO}^{2}$ & $0,13(0,01)$ & 0,34 & $1,92(0,09)$ & 1,84 & $0,44(0,02)$ & 0,56 \\
\hline $\mathrm{MgO}$ & $1,11(0,02)$ & 1,42 & $2,09(0,06)$ & 1,91 & $1,55(0,05)$ & 1,64 \\
\hline $\mathrm{Na}_{2} \mathrm{O}$ & $0,26(0,06)$ & 0,32 & $1,95(0,22)$ & 1,80 & $0,90(0,11)$ & 1,16 \\
\hline $\mathrm{K}_{2} \mathrm{O}^{2}$ & $2,31(0,11)$ & 2,77 & $2,88(0,13)$ & 3,00 & $2,98(0,21)$ & 3,16 \\
\hline
\end{tabular}

\section{AGRADECIMENTOS}

Ao $\mathrm{CNPq}$ pelas bolsas e apoio à pesquisa concedidos (Processo 460.822/00-4) e à FAPESP (Processo 98/10814-3).

\section{REFERÊNCIAS}

1. Ingle Jr, J. D.; Crouch, S. R.; Spectrochemical Analysis, Prentice Hall, Englewood Cliffs: New Jersey, 1988

2. Kurfürst, U., ed.; Solid Sample Analysis - Direct and Slurry Sampling Using GF-AAS and ETV-ICP, Springer-Verlag: Berlim, 1998.

3. Greenfield, S.; Montaser, A. Em Inductively Coupled Plasmas in Analytical Atomic Spectrometry, Montaser, A.; Golightly, D. W., eds; $2^{\text {nd }}$ ed., VCH: New York, 1992, pp. 187-247.

4. Ebdon, L.; Foukes, M.; Sutton, K.; J. Anal. At. Spectrom. 1997, 12, 213.
5. Broekaert, J. A. C.; Lathen, C.; Brandt, R.; Pilger, C.; Pollmann, D., Tschöpel, P.; Tölg, G.; Fresenius' J. Anal. Chem. 1994, 349, 20.

6. Fuller, C. W.; Hutton, R. C.; Preston, B.; Analyst 1981, 106, 913.

7. Browner, R. F.; Boorn, A. W.; Anal. Chem. 1984, 56, A786.

8. Brenner, I. B.; Zander, A. T.; Spectrochim. Acta, Part B 2000, 55, 1195.

9. Garcia, E. E.; Nogueira, A. R. A.; Nóbrega, J. A.; J. Anal. At. Spectrom. 2001, 16, 825 .

10. Silva, C. S.; Blanco, T.; Nóbrega, J. A.; Spectrochim. Acta, Part B 2002, $57,29$.

11. Laird, D. A.; Dowdy, R. H.; Munter, R. C.; J. Anal. At. Spectrom. 1990, 5,515 .

12. Ma Z.; Merkus, H. G.; de Smet, J. G. A. F.; Heffels, C.; Scarlett, B.; Powder Technol. 2000, 111, 66.

13. Sulcek, Z.; Povondra, P.; Methods of Decomposition in Inorganic Analysis. CRC Press: Flórida, 1992.

14. Brenner, I. B.; comunicação pessoal. 